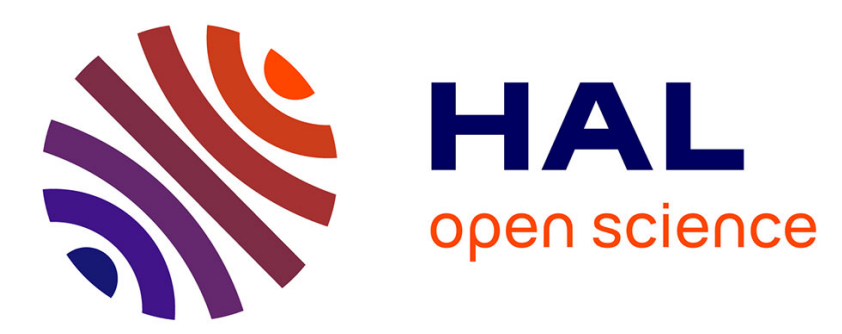

\title{
Computing necessary integrability conditions for planar parametrized homogeneous potentials
}

Alin Bostan, Thierry Combot, Mohab Safey El Din

\section{To cite this version:}

Alin Bostan, Thierry Combot, Mohab Safey El Din. Computing necessary integrability conditions for planar parametrized homogeneous potentials. ISSAC'14 - International Symposium on Symbolic and Algebraic Computation, Jul 2014, Kobe, Japan. pp.67-74, 10.1145/2608628.2608662 . hal-00994116

\section{HAL Id: hal-00994116 https://hal.inria.fr/hal-00994116}

Submitted on 21 May 2014

HAL is a multi-disciplinary open access archive for the deposit and dissemination of scientific research documents, whether they are published or not. The documents may come from teaching and research institutions in France or abroad, or from public or private research centers.
L'archive ouverte pluridisciplinaire $\mathbf{H A L}$, est destinée au dépôt et à la diffusion de documents scientifiques de niveau recherche, publiés ou non, émanant des établissements d'enseignement et de recherche français ou étrangers, des laboratoires publics ou privés. 


\section{Computing necessary integrability conditions for planar parametrized homogeneous potentials* \\ Alin Bostan
INRIA (France) \\ Alin.Bostan@inria.fr \\ Thierry Combot \\ Institut de Mathématiques de \\ Bourgogne UMR CNRS 5584 \\ Univ. de Bourgogne (France) \\ thierry.combot@u-bourgogne.fr

\author{
Mohab Safey El Din \\ Sorbonne Universities \\ Univ. Pierre et Marie Curie \\ (Paris 06) \\ INRIA Paris Rocquencourt, \\ POLSYS Project \\ LIP6 CNRS, UMR 7606 \\ Mohab.Safey@lip6.fr
} \\ Institut Universitaire de France}

\begin{abstract}
Let $V \in \mathbb{Q}(i)\left(\mathbf{a}_{1}, \ldots, \mathbf{a}_{n}\right)\left(\mathbf{q}_{1}, \mathbf{q}_{2}\right)$ be a rationally parametrized planar homogeneous potential of homogeneity degree $k \neq-2,0,2$. We design an algorithm that computes polynomial necessary conditions on the parameters $\left(\mathbf{a}_{1}, \ldots, \mathbf{a}_{n}\right)$ such that the dynamical system associated to the potential $V$ is integrable. These conditions originate from those of the Morales-Ramis-Simó integrability criterion near all Darboux points. The implementation of the algorithm allows to treat applications that were out of reach before, for instance concerning the nonintegrability of polynomial potentials up to degree 9 . Another striking application is the first complete proof of the non-integrability of the collinear three body problem.
\end{abstract}

\section{Categories and Subject Descriptors:}

I.1.2 [Computing Methodologies]: Symbolic and Algebraic Manipulations - Algebraic Algorithms

General Terms: Algorithms, Theory.

Keywords: Integrability, potentials, algorithms.

\section{INTRODUCTION}

Let us consider the Hamiltonian system

$$
\dot{\mathbf{q}}_{1}=\mathbf{p}_{1}, \quad \dot{\mathbf{q}}_{2}=\mathbf{p}_{2}, \quad \dot{\mathbf{p}}_{1}=-\frac{\partial V}{\partial \mathbf{q}_{1}}, \quad \dot{\mathbf{p}}_{2}=-\frac{\partial V}{\partial \mathbf{q}_{2}}
$$

with $V \in \mathbb{C}\left(\mathbf{q}_{1}, \mathbf{q}_{2}\right)$, called the potential. System (1) describes the motion of a particle in the plane submitted to the force field $\nabla V(\mathbf{q})$. It always admits the so-called Hamiltonian $H=\frac{1}{2}\left(\mathbf{p}_{1}^{2}+\mathbf{p}_{2}^{2}\right)+V\left(\mathbf{q}_{1}, \mathbf{q}_{2}\right)$ as a rational first integral. The potential $V$ is called (rationally) integrable if system (1) admits another rational first integral $I$, functionally independent on $H$. Intuitively, the integrability of $V$ is equivalent to the fact that (1) can be solved in explicit terms.

Integrability is a rare phenomenon and it is in general a difficult task to determine whether a given potential is integrable or not. For homogeneous potentials in $\mathbb{C}\left(\mathbf{q}_{1}, \mathbf{q}_{2}\right)$, necessary conditions for integrability were given by Morales-Ramis [19] and by Morales-RamisSimó [22]. Building on these works, we design in this article an algorithm which takes as input a family of rational homogeneous potentials $V \in \mathbb{Q}(i)(\mathbf{a})\left(\mathbf{q}_{1}, \mathbf{q}_{2}\right)$ depending on parameters $\mathbf{a}=\left(\mathbf{a}_{1}, \ldots, \mathbf{a}_{n}\right)$

\footnotetext{
*We thank the referees for their helpful comments. This work has been supported in part by the Microsoft Research - Inria Joint Centre.

Permission to make digital or hard copies of all or part of this work for personal or classroom use is granted without fee provided that copies are not made or distributed for profit or commercial advantage and that copies bear this notice and the full citation on the first page. Copyrights for components of this work owned by others than ACM must be honored. Abstracting with credit is permitted. To copy otherwise, or republish, to post on servers or to redistribute to lists, requires prior specific permission and/or a fee. Request permissions from Permissions@acm.org.

ISSAC '14, July 21 - 25, 2014, Kobe, Japan.

Copyright 2014 ACM 978-1-4503-2501-1/14/07 ...\$15.00.

http://dx.doi.org/10.1145/2608628.2608662.
}

and which computes a set of constraints on the parameter values $a \in \mathbb{C}^{n}$ that are necessary for the integrability of $V\left(a, \mathbf{q}_{1}, \mathbf{q}_{2}\right)$. These constraints turn out to be of polynomial nature in $\mathbf{a}$.

There are several difficulties in this parameterized setting. The first one is that the integrability constraints provided by the MoralesRamis theory —on which our whole approach relies—-, are expressed in terms of quantities (eigenvalues of Hessian matrices at Darboux points, see Section 2) which are not easily accessible. We circumvent this basic difficulty by using an equation that relates the eigenvalues, but this brings a new technical complication since the equation is of Diophantine type. A third difficulty is that the number of Darboux points itself may depend on the parameters, leading to singular cases.

We follow a classical approach, inspired mostly by ideas in [14]. Our contribution to the topic is effective and algorithmic, as we provide a complete, proven and implemented algorithm for the problem of computing necessary integrability conditions for planar parametrized homogeneous potentials, with precise output specifications. Our algorithm uses classical tools in computer algebra, such as polynomial ideal elimination based on Gröbner bases techniques. An important feature is the use of (complex) polar coordinates to represent homogeneous potentials by univariate rational functions with parameters $F \in \mathbb{Q}(i)(\mathbf{a})(\mathbf{z})$. This change of representation considerably simplifies the computations and the proofs. For instance, in polar representation, singular cases are those with non-generic multiplicity of the roots/poles of $F$. They are treated by our algorithm, which builds a tree containing each possible singular case. This approach is related with comprehensive Gröbner bases [31], which are avoided here thanks to some a priori knowledge about singular cases.

In summary, our strategy for computing necessary integrability conditions for $V$ consists in 4 steps: $(i)$ rewrite $V$ in polar coordinates; (ii) set up a Diophantine equation whose solutions belong to the socalled Morales-Ramis table (that contains all possible eigenvalues of the Hessian of $V$ at Darboux points of $V$ ); (iii) solve this Diophantine equation; (iv) rewrite the condition of having prescribed eigenvalues at Darboux points as polynomial conditions on $\mathbf{a}$.

Some prior works used a similar strategy, but it was unclear which cases were possible to tackle, in particular for singular ones. The approach was not fully automatized and this explains that results were only available for special families of potentials, for instance polynomials of small degree (3 or 4$)[13,14,11,12]$, as the number of singular cases grows very fast (already 44 for polynomials of degree 5). By contrast, our treatment is unified and fully automated, and it allows not only to retrieve (and sometimes correct) known results, but more importantly, to treat potentials of degrees previously unreached (up to 9). By applying our algorithm to polynomial potentials, we found three new cases admissible for integrability at degree 5 (but still not proved to be integrable), and various new families for higher degrees. An even more striking application of our algorithm is the first complete proof of the non-integrability of the collinear three body problem, on which only partial results were known [32, 23, 27]. The direct approach that consists in searching first integrals $[8,24]$ is complementary to our (non-)integrability analysis, as our algorithm helps either proving that the lists in $[8,24]$ are complete, or finding new 
unknown cases.

Warning: We will assume throughout the article that the homogeneity degree $k$ is different from $-2,0$ and 2 . (This is because the Morales-Ramis theory is much less powerful when $k \in\{-2,0,2\}$.)

Convention of notation: to avoid confusion, we will use bold letters for variables/parameters, and italic letters for parameter values.

\section{PRELIMINARIES AND BASIC NOTIONS}

There exist strong integrability constraints (see Theorem 1 below). They require to deal with Darboux points, whose definition we now recall.

Definition 1 Let $V \in \mathbb{C}\left(\mathbf{q}_{1}, \mathbf{q}_{2}\right)$ be a homogeneous rational function of homogeneity degree $k \neq 0$. A point $c=\left(c_{1}, c_{2}\right) \in \mathbb{C}^{2} \backslash\{0\}$ is called $a$ (proper) Darboux point of $V$ if it satisfies the equations

$$
\frac{\partial V}{\partial \mathbf{q}_{1}}(\mathbf{c})=k \mathbf{c}_{1}, \quad \frac{\partial V}{\partial \mathbf{q}_{2}}(\mathbf{c})=k \mathbf{c}_{2} \text {. }
$$

Note that, by homogeneity, we could have chosen an arbitrary normalization non-zero constant on the right-hand side of (2). In the literature, this normalization constant is frequently chosen equal to 1 [21]. However, our choice is deliberate, see the remark after Theorem 1.

The following result (which is an application of a more general criterion due to Morales and Ramis [19]) provides necessary conditions for integrability under the form of constraints on eigenvalues of Hessian matrices at each Darboux point. It is the basic ingredient for numerous non-integrability proofs $[13,14,23,20,29,15,17,25$, 1]. Roughly, its main idea is as follows. A Darboux point leads to a straight line orbit of the dynamical system (1) associated to $V$, around which the system (1) can be linearized. If the whole system is integrable, then the linearized system, which in our case corresponds to a hypergeometric equation, is also integrable. Thus the integrability table of Theorem 1 below is reminiscent of Kimura's classification [10] of solvable hypergeometric equations.

Theorem 1 (Morales-Ramis [21]) Let $V \in \mathbb{C}\left(\mathbf{q}_{1}, \mathbf{q}_{2}\right)$ be a homogeneous rational function of homogeneity degree $k \neq-2,0,2$, and let $c \in \mathbb{C}^{2} \backslash\{0\}$ be a Darboux point of $V$. If the potential $V$ is integrable, then for any eigenvalue $\lambda$ of the Hessian matrix of $V$ at $c$, the pair $(k, \lambda)$ belongs to the following table, for some $j \in \mathbb{Z}$.

\begin{tabular}{|c|c||c|c|}
\hline$k$ & $\lambda$ & $k$ & $\lambda$ \\
\hline $\mathbb{Z}^{*}$ & $\frac{1}{2} j k(j k+k-2)$ & -3 & $-\frac{25}{8}+\frac{1}{8}\left(\frac{12}{5}+6 j\right)^{2}$ \\
\hline $\mathbb{Z}^{*}$ & $\frac{1}{2}(j k+1)(j k+k-1)$ & 3 & $-\frac{1}{8}+\frac{1}{8}(2+6 j)^{2}$ \\
\hline-5 & $-\frac{49}{8}+\frac{1}{8}\left(\frac{10}{3}+10 j\right)^{2}$ & 3 & $-\frac{1}{8}+\frac{1}{8}\left(\frac{3}{2}+6 j\right)^{2}$ \\
\hline-5 & $-\frac{49}{8}+\frac{1}{8}(4+10 j)^{2}$ & 3 & $-\frac{1}{8}+\frac{1}{8}\left(\frac{6}{5}+6 j\right)^{2}$ \\
\hline-4 & $-\frac{9}{2}+\frac{1}{2}\left(\frac{4}{3}+4 j\right)^{2}$ & 3 & $-\frac{1}{8}+\frac{1}{8}\left(\frac{12}{5}+6 j\right)^{2}$ \\
\hline-3 & $-\frac{25}{8}+\frac{1}{8}(2+6 j)^{2}$ & 4 & $-\frac{1}{2}+\frac{1}{2}\left(\frac{4}{3}+4 j\right)^{2}$ \\
\hline-3 & $-\frac{25}{8}+\frac{1}{8}\left(\frac{3}{2}+6 j\right)^{2}$ & 5 & $-\frac{9}{8}+\frac{1}{8}\left(\frac{10}{3}+10 j\right)^{2}$ \\
\hline-3 & $-\frac{25}{8}+\frac{1}{8}\left(\frac{6}{5}+6 j\right)^{2}$ & 5 & $-\frac{9}{8}+\frac{1}{8}(4+6 j)^{2}$ \\
\hline
\end{tabular}

This table will be called throughout the article the Morales-Ramis table. For a fixed homogeneity degree $k$, we will denote by $E_{k}$ the infinite set of allowed eigenvalues $\lambda$ in the table, corresponding to $k$.

Note two differences with the classical statement of the MoralesRamis theorem. First, due to our choice of the normalization constant in Definition 1 ( $k$ instead of 1 ), the eigenvalues displayed in the previous table are $k$ times larger than those of [21, Theorem 3]. Our choice is motivated by the fact that it simplifies the computations, and it has the nice and useful property that the eigenvalue sets in the table are lower bounded. Second, both the original proof [21] and the statement of the Morales-Ramis theorem [6, Theorem 1.2], require the additional assumption that the Hessian matrix of $V$ at $c$ is diagonalizable; but in fact, [6, Theorem 1.3(1)] shows that this hypothesis is not necessary.
We now illustrate the basic notion of Darboux points and the use of Theorem 1 on a toy parametrized example. The example is simple enough so that the eigenvalues are accessible by a direct computation.

Example Consider the homogeneous potential

$$
V\left(\mathbf{a}, \mathbf{q}_{1}, \mathbf{q}_{2}\right)=\left(\mathbf{a}_{1} \mathbf{q}_{1}+\mathbf{a}_{2} \mathbf{q}_{2}\right)\left(\mathbf{q}_{1}^{2}+\mathbf{q}_{2}^{2}\right) \text {. }
$$

The homogeneity degree is $k=3$ and the Darboux point equation (2) is

$$
3 \mathbf{a}_{1} \mathbf{c}_{1}^{2}+\mathbf{a}_{1} \mathbf{c}_{2}^{2}+2 \mathbf{a}_{2} \mathbf{c}_{1} \mathbf{c}_{2}=3 \mathbf{c}_{1}, 3 \mathbf{a}_{2} \mathbf{c}_{2}^{2}+\mathbf{a}_{2} \mathbf{c}_{1}^{2}+2 \mathbf{a}_{1} \mathbf{c}_{1} \mathbf{c}_{2}=3 \mathbf{c}_{2} .
$$

For parameter values $\left(a_{1}, a_{2}\right) \in \mathbb{C}^{2}$ such that $a_{1}^{2}+a_{2}^{2} \neq 0$, its solutions $c=\left(c_{1}, c_{2}\right) \in \mathbb{C}^{2} \backslash\{0\}$ read

$$
c=\left(\frac{a_{1}}{a_{1}^{2}+a_{2}^{2}}, \frac{a_{2}}{a_{1}^{2}+a_{2}^{2}}\right), \quad c=\left(\frac{3}{2\left(a_{1} \pm i a_{2}\right)}, \frac{ \pm 3 i}{2\left(a_{1} \pm i a_{2}\right)}\right) \text {. }
$$

The Hessian matrices at these points are

$$
\left(\begin{array}{cc}
\frac{2\left(3 a_{1}^{2}+a_{2}^{2}\right)}{a_{1}^{2}+a_{2}^{2}} & \frac{4 a_{1} a_{2}}{a_{1}^{2}+a_{2}^{2}} \\
\frac{4 a_{1} a_{2}}{a_{1}^{2}+a_{2}^{2}} & \frac{2\left(a_{1}^{2}+3 a_{2}^{2}\right)}{a_{1}^{2}+a_{2}^{2}}
\end{array}\right),\left(\begin{array}{cc}
\frac{3\left(3 a_{1} \pm i a_{2}\right)}{a_{1} \pm i a_{2}} & \frac{3\left(a_{2} \pm i a_{1}\right)}{a_{1} \pm i a_{2}} \\
\frac{3\left(a_{2} \pm i a_{1}\right)}{a_{1} \pm i a_{2}} & \frac{3\left(a_{1} \pm 3 i a_{2}\right)}{a_{1} \pm i a_{2}}
\end{array}\right) .
$$

The eigenvalues of the first matrix are $\{6,2\}$. The (a priori unexpected) fact that none of them depend on the parameter values $a_{1}, a_{2}$ comes from a relation on eigenvalues at Darboux points that will be proved later (Theorem 3). Now, Theorem 1 tells us that $E_{3}$, the set of allowed eigenvalues for homogeneity degree $k=3$, is the set of the rational numbers of the form

$$
\begin{array}{r}
\frac{3}{2} j(3 j+1), \frac{1}{2}(3 j+1)(3 j+2),-\frac{1}{8}+\frac{1}{8}\left(6 j+\frac{12}{5}\right)^{2}, \\
-\frac{1}{8}+\frac{1}{8}\left(6 j+\frac{3}{2}\right)^{2},-\frac{1}{8}+\frac{1}{8}\left(6 j+\frac{6}{5}\right)^{2},-\frac{1}{8}+\frac{1}{8}(6 j+2)^{2},
\end{array}
$$

where $j \in \mathbb{Z}$. The eigenvalue $\lambda=6$ is allowed (by choosing $j=1$ in the first sequence), but the eigenvalue $\lambda=2$ is not. This can be seen by simply solving for integers six quadratic equations. Thus, by Theorem 1 , the potential $V\left(a_{1}, a_{2}, \mathbf{q}_{1}, \mathbf{q}_{2}\right)$ is not integrable when $a_{1}^{2}+a_{2}^{2} \neq 0$, and a necessary condition for integrability is $a_{1}^{2}+a_{2}^{2}=0$.

\section{POLAR REPRESENTATION}

We will use complex polar coordinates in order to represent a given rational homogeneous potential $V$ in a simpler way, by a pair $(F, k)$, where $F$ is a univariate rational function, and $k$ is an integer. In this new representation, various quantities attached to $V$, such as Darboux points and eigenvalues of the Hessian of $V$, are much easier to express, including a useful relation (11) on these eigenvalues. This representation has already been used for non-integrability proofs $[26,16$, 30]. This section provides an overview on some results on polar coordinates with useful properties needed to prove our algorithm (see Theorem 2 below).

In the rest of the article, we will use the notation $\Delta$ and $D$ for the following subdomains of $\mathbb{C}^{2}$ :

$$
\begin{aligned}
& \Delta=\mathbb{C}^{*} \times\{\theta \in \mathbb{C}, 0 \leq \operatorname{Re}(\theta)<2 \pi\}, \\
& D=\left\{\left(q_{1}, q_{2}\right) \in \mathbb{C}^{2}, q_{1}^{2}+q_{2}^{2} \neq 0\right\},
\end{aligned}
$$

and $\varphi$ for the map $\varphi: \Delta \rightarrow D$ defined by $\varphi(r, \theta)=(r \cos \theta, r \sin \theta)$.

Proposition 1 The map $\varphi$ is differentiable on $\Delta$, and its image is a double covering of $D$ (i.e., each fiber $\varphi^{-1}\left(q_{1}, q_{2}\right)$ contains exactly two points).

PRoOF. The functions $(r, \theta) \mapsto r \cos \theta$ and $(r, \theta) \mapsto r \sin \theta$ are differentiable on $\Delta$, and thus $\varphi$ is differentiable on $\Delta$. The relation $(r \cos \theta)^{2}+(r \sin \theta)^{2}=r^{2}$ implies that the image of $\varphi$ is contained 
in $D$. Let us compute the inverse of $\varphi$. If $q_{1}=r \cos \theta$ and $q_{2}=r \sin \theta$ with $(r, \theta) \in \Delta$, then $r^{2}=q_{1}^{2}+q_{2}^{2}$ and $e^{i \theta}=\left(q_{1}+i q_{2}\right) / r$. The first relation determines $r$ up to a sign; since $q_{1}^{2}+q_{2}^{2} \neq 0$ there are always exactly two possible choices $\pm r$. After this sign choice, $e^{i \theta}$ is uniquely determined, thus $\theta$ is determined up to translation by $2 \pi$. Since $0 \leq \operatorname{Re}(\theta)<2 \pi$, then $\theta$ is uniquely determined.

Proposition 2 Any homogeneous potential $V \in \mathbb{C}\left(\mathbf{q}_{1}, \mathbf{q}_{2}\right)$ can be written in complex polar coordinates

$$
V\left(q_{1}, q_{2}\right)=r^{k} F\left(e^{i \theta}\right), \quad \text { for } \quad\left(q_{1}, q_{2}\right)=\varphi(r, \theta),
$$

where $k$ is the homogeneity degree of $V$, and $F$ is a rational function in $\mathbb{C}(\mathbf{z})$ having the same parity as $k$.

Moreover, if $V \in \mathbb{Q}\left(\mathbf{q}_{1}, \mathbf{q}_{2}\right)$, then $F$ belongs to $\mathbb{Q}(i)(\mathbf{z})$.

Proof. Let $F$ be the rational function in $\mathbb{C}(\mathbf{z})$ defined by

$$
F(\mathbf{z})=V\left(\frac{\mathbf{z}+\mathbf{z}^{-1}}{2}, \frac{\mathbf{z}-\mathbf{z}^{-1}}{2 i}\right) .
$$

Then $F\left(e^{i \theta}\right)$ is equal to $V(\cos \theta, \sin \theta)$, and homogeneity of $V$ allows to conclude that for $q_{1}=r \cos (\theta)$ and $q_{2}=r \sin (\theta)$ we have

$$
V\left(q_{1}, q_{2}\right)=r^{k} V(\cos \theta, \sin \theta)=r^{k} F\left(e^{i \theta}\right) .
$$

Using again that $V$ is $k$-homogeneous, we obtain that

$$
F(-\mathbf{z})=V\left(-\frac{\mathbf{z}+\mathbf{z}^{-1}}{2},-\frac{\mathbf{z}-\mathbf{z}^{-1}}{2 i}\right)=(-1)^{k} F(\mathbf{z}),
$$

and thus $F$ has the same parity as $k$. The last assertion is obvious by definition of $F$.

Proposition 2 shows that the homogeneous rational potential $V$ is represented in polar coordinates by a pair $(F, k)$, where $F$ is a univariate rational function, and $k$ is an integer. We now write the equation of a Darboux point $c \in D$ of a potential $V$ and the eigenvalues of the corresponding Hessian matrix $\nabla^{2} V(c)$ in polar coordinates.

Proposition 3 Let $V \in \mathbb{C}\left(\mathbf{q}_{1}, \mathbf{q}_{2}\right)$ be a homogeneous potential with polar representation $(F, k)$, and let $c=\left(c_{1}, c_{2}\right) \in D$ be a Darboux point for $V$. Then for $(r, \theta) \in \varphi^{-1}(c)$ we have

$$
F^{\prime}\left(e^{i \theta}\right)=0 \quad \text { and } \quad F\left(e^{i \theta}\right)=r^{2-k} .
$$

Moreover, $\left(c_{1}, c_{2}\right)^{t}$ and $\left(-c_{2}, c_{1}\right)^{t}$ are eigenvectors of the Hessian matrix $\nabla^{2} V(c)$, with respective eigenvalues

$$
k(k-1) \quad \text { and } \quad k-\frac{e^{2 i \theta} F^{\prime \prime}\left(e^{i \theta}\right)}{F\left(e^{i \theta}\right)} .
$$

PROOF. We start from the relations

$$
V\left(\mathbf{q}_{1}, \mathbf{q}_{2}\right)=\mathbf{r}^{k} F(\mathbf{z}), \quad \mathbf{r}^{2}=\mathbf{q}_{1}^{2}+\mathbf{q}_{2}^{2}, \quad \mathbf{z}=\frac{\mathbf{q}_{1}+i \mathbf{q}_{2}}{\mathbf{r}}=e^{i \theta} .
$$

From there we deduce, by differentiation, the equalities

$$
\frac{\partial \mathbf{r}}{\partial \mathbf{q}_{1}}=\frac{\mathbf{q}_{1}}{\mathbf{r}}, \quad \frac{\partial \mathbf{r}}{\partial \mathbf{q}_{2}}=\frac{\mathbf{q}_{2}}{\mathbf{r}}, \quad \frac{\partial \mathbf{z}}{\partial \mathbf{q}_{1}}=\frac{-i \mathbf{q}_{2} \mathbf{z}}{\mathbf{r}^{2}}, \quad \frac{\partial \mathbf{z}}{\partial \mathbf{q}_{2}}=\frac{i \mathbf{q}_{1} \mathbf{z}}{\mathbf{r}^{2}}
$$

These equalities imply that the derivatives of $V$ write

$$
\begin{aligned}
& \frac{\partial V}{\partial \mathbf{q}_{1}}=\mathbf{r}^{k-2}\left(k \mathbf{q}_{1} F(\mathbf{z})-i \mathbf{q}_{2} \mathbf{z} F^{\prime}(\mathbf{z})\right), \\
& \frac{\partial V}{\partial \mathbf{q}_{2}}=\mathbf{r}^{k-2}\left(k \mathbf{q}_{2} F(\mathbf{z})+i \mathbf{q}_{1} \mathbf{z} F^{\prime}(\mathbf{z})\right) .
\end{aligned}
$$

Combining these last two equations yields

$$
\mathbf{q}_{1} \frac{\partial V}{\partial \mathbf{q}_{1}}+\mathbf{q}_{2} \frac{\partial V}{\partial \mathbf{q}_{2}}=k V, \quad \mathbf{q}_{1} \frac{\partial V}{\partial \mathbf{q}_{2}}-\mathbf{q}_{2} \frac{\partial V}{\partial \mathbf{q}_{1}}=i \mathbf{r}^{k} \mathbf{z} F^{\prime}(\mathbf{z})
$$

(The first one is Euler's relation for $k$-homogeneous functions.) Evaluating equalities (8) at the Darboux point $c$, and using the Darboux point equation (2), yields the proof of (4).
Let us now prove the last assertion of the proposition. By differentiating the first equality in (8) with respect to $\mathbf{q}_{1}$ and $\mathbf{q}_{2}$, by evaluating at $c$, and by using (4), we obtain $\nabla^{2} V(c) \cdot c^{t}+k c^{t}=k^{2} c^{t}$. Thus $c^{t}$ is an eigenvector of $\nabla^{2} V(c)$, with corresponding eigenvalue $k(k-1)$.

Similarly, differentiating the second equality in (8) and specializing the result at $c$ yields $\nabla^{2} V(c) \cdot v-k v=-r^{k-2} e^{2 i \theta} F^{\prime \prime}\left(e^{i \theta}\right) \cdot v$, where $v$ denotes the vector $\left(-c_{2}, c_{1}\right)^{t}$. This concludes the proof.

Proposition 3 motivates the following definition of Darboux points in polar representation, and of associated eigenvalues.

Definition 2 Let $(F, k)$ be the polar representation of a homogeneous potential $V \in \mathbb{C}\left(\mathbf{q}_{1}, \mathbf{q}_{2}\right)$. A complex number $z \in \mathbb{C} \backslash\{0\}$ is called a Darboux point of $F$ if $F^{\prime}(z)=0$ and $F(z) \neq 0$. A Darboux point $z \in \mathbb{C} \backslash\{0\}$ is said to be multiple if $z$ is a multiple root of $F^{\prime}$; else it is said to be simple.

If $z \in \mathbb{C} \backslash\{0\}$ is a Darboux point for $F$, we will call associated eigenvalues the values $k(k-1)$ and $k-z^{2} F^{\prime \prime}(z) / F(z)$.

The map $\varphi$ naturally sends Darboux points in polar representation to Darboux points in Cartesian coordinates in $D$, also carrying the definition of associated eigenvalues.

We now prove the main result of this subsection; it gives a necessary condition for integrability of a homogeneous potential in terms of its polar representation. We recall that $E_{k}$ is the set of allowed values in the Morales-Ramis table for degree $k$.

Theorem 2 Let $V \in \mathbb{C}\left(\mathbf{q}_{1}, \mathbf{q}_{2}\right)$ be a homogeneous potential with polar representation $(F, k)$ and let $\Lambda$ be the following set

$$
\Lambda(F, k):=\left\{k-\frac{z^{2} F^{\prime \prime}(z)}{F(z)} \mid z \neq 0, F^{\prime}(z)=0, F(z) \neq 0\right\} .
$$

Let $\operatorname{Sp}_{D}\left(\nabla^{2} V\right)$ denote the union of the sets $\operatorname{Sp}\left(\nabla^{2} V(c)\right)$ taken over all Darboux points $c \in D$ of $V$. Then

$$
\{k(k-1)\} \cup \operatorname{Sp}_{D}\left(\nabla^{2} V\right)=\{k(k-1)\} \cup \Lambda .
$$

Moreover, if $V$ is integrable, then $\Lambda \subseteq E_{k}$.

PROOF. We first prove equality (10). Proposition 3 readily yields the inclusion $\{k(k-1)\} \cup \operatorname{Sp}_{D}\left(\nabla^{2} V\right) \subseteq\{k(k-1)\} \cup \Lambda$. Indeed, if $\lambda$ is in $\operatorname{Sp}_{D}\left(\nabla^{2} V\right) \backslash\{k(k-1)\}$, then there exists a Darboux point $c \in D$ of $V$ such that $\lambda \in \operatorname{Sp}\left(\nabla^{2} V(c)\right) \backslash\{k(k-1)\}$. Then letting $(r, \theta) \in$ $\varphi^{-1}(c)$, Proposition 3 implies that $z=e^{i \theta}$ satisfies $z \neq 0, F^{\prime}(z)=0$, (5) $F(z) \neq 0$ and $\operatorname{Sp}\left(\nabla^{2} V(c)\right)=\left\{k(k-1), k-z^{2} F^{\prime \prime}(z) / F(z)\right\}$. Therefore, $\lambda$ is equal to $k-z^{2} F^{\prime \prime}(z) / F(z)$, and thus it belongs to $\Lambda$.

Conversely, let $\lambda$ be in $\Lambda \backslash\{k(k-1)\}$. There exists a $z \in \mathbb{C} \backslash\{0\}$ such that $F^{\prime}(z)=0, F(z) \neq 0$ and $\lambda=k-z^{2} F^{\prime \prime}(z) / F(z)$. Write this $z$ as $e^{i \theta}$ for some $\theta$ with $\operatorname{Re}(\theta) \in[0,2 \pi)$, and write $F(z)$ as $r^{2-k}$ with $r \in \mathbb{C}^{*}$. Then, Equations (6) and (7) imply that $c=\varphi(r, \theta)$ is a Darboux point of $V$ in $D$. By Proposition 3, $\lambda$ belongs to $\operatorname{Sp}_{D}\left(\nabla^{2} V\right)$. Equality (10) is now proven.

To prove the last assertion, assume that $V$ is integrable. Then Theorem 1 shows that $\operatorname{Sp}_{D}\left(\nabla^{2} V\right) \subseteq E_{k}$. Since the eigenvalue $k(k-1)$ belongs to the Morales-Ramis table (first sequence with $j=1$ ), we also

(6) have $\{k(k-1)\} \cup \operatorname{Sp}_{D}\left(\nabla^{2} V\right) \subseteq E_{k}$. The desired inclusion $\Lambda \subseteq E_{k}$ is then a consequence of equality (10).

\section{A SPECIAL DIOPHANTINE EQUATION}

\subsection{Sets of possible eigenvalues}

There are infinitely many allowed eigenvalues for integrability in the Morales-Ramis table. We now prove an interesting equation that relates the eigenvalues of the Hessian matrix of $V$ at Darboux points. This will allow us to bound the possible eigenvalues allowed for integrability. 
Definition 3 For a rational function $F \in \mathbb{C}(\mathbf{z}) \backslash\{0\}$, we denote by $k_{0}=k_{0}(F)$ and $k_{\infty}=k_{\infty}(F) \in \mathbb{Z}$ two integers such that $F(\mathbf{z}) \underset{z \rightarrow 0}{\sim} a_{0} \mathbf{z}^{k_{0}}$ and $F(\mathbf{z}) \underset{z \rightarrow \infty}{\sim} a_{\infty} \mathbf{z}^{k_{\infty}}$, with $a_{0}, a_{\infty} \in \mathbb{C}(\mathbf{z}) \backslash\{0\}$. These integers will be called asymptotic exponents of $F$ (at zero and infinity).

Theorem 3 Let $V \in \mathbb{C}\left(\mathbf{q}_{1}, \mathbf{q}_{2}\right) \backslash\{0\}$ be a homogeneous potential with polar representation $(F, k)$, let $\Lambda$ be the set defined in (9) (Theorem 2$)$, counting multiplicities, and $k_{0}, k_{\infty} \in \mathbb{Z}$ be the asymptotic exponents of $F$. If $k_{0} k_{\infty} \neq 0$ and if $F$ has only simple Darboux points, then:

$$
\sum_{\lambda \in \Lambda} \frac{1}{\lambda-k}=\frac{1}{k_{0}}-\frac{1}{k_{\infty}} .
$$

This result is a generalization of [14, Theorem 2.3] for polynomial homogeneous potentials, and was already proved under an equivalent form in [28, Theorem 1.7]. Still, in [28], the polar representation is not used, leading to a more complicated description of the set $\Lambda$, a less readable relation (11) (but not harder to compute in practice) and a more complicated proof. This is why we display here a simple self-contained proof of Theorem 3.

Proof. Consider the rational function $T(\mathbf{z})=\mathbf{z}^{-2} F(\mathbf{z}) / F^{\prime}(\mathbf{z})$. We study its poles. Since $F(\mathbf{z}) \underset{z \rightarrow 0}{\sim} a_{0} \mathbf{z}^{k_{0}}$ with $k_{0} \neq 0$, the origin is a pole of $T$. Moreover, the power series expansion of $T$ at $z=0$ gives $T(\mathbf{z})=1 /\left(k_{0} \mathbf{z}\right)+o(1)$, which shows that $z=0$ is a pole of order 1 of $T$, with residue $1 / k_{0}$. Similarly, $z=\infty$ is a pole of order 1 of $T$ with residue $1 / k_{\infty}$

Let now $z_{0} \neq 0$ be a finite pole of $T$. It is either a pole of $F$, or a root of $F^{\prime}$. Any nonzero pole of $F$ of order $j$ is a pole of $F^{\prime}$ of order $j+1$, and thus it is not a pole of $T$. Thus, $z_{0}$ is necessarily a root of $F^{\prime}$. By the assumption that all Darboux points of $F$ are simple, $z_{0}$ is not a root of $F^{\prime \prime}$. Therefore, $z_{0}$ is a pole of $T$ of order 1 , and the series expansion $F^{\prime}(\mathbf{z})=F^{\prime \prime}\left(z_{0}\right)\left(\mathbf{z}-z_{0}\right)+o\left(\left(\mathbf{z}-z_{0}\right)^{2}\right)$ shows that the residue of $T$ at $z_{0}$ is $F\left(z_{0}\right) /\left(z_{0}^{2} F^{\prime \prime}\left(z_{0}\right)\right)$. Recognizing this expression as $1 /(k-\lambda)$ for some $\lambda \in \Lambda$, and using Cauchy's residue formula, proves Equation (11).

Theorem 3 contains two assumptions, that $k_{0} k_{\infty} \neq 0$ and that Darboux points of $F$ are simple. The first assumption does not always hold, and then the possible eigenvalues could be unbounded, as proven for instance by a family of potentials in [3], for which stronger integrability conditions were needed (there is a similar difficulty in [18]). The second hypothesis (simple Darboux points) is not always satisfied, but [2, Theorem 1] provides a classification of integrable potentials with a multiple Darboux point: they are invariant by rotation, i.e. with $F$ constant. This motivates the following definition.

Definition 4 We say that a homogeneous potential $V \in \mathbb{C}\left(\mathbf{q}_{1}, \mathbf{q}_{2}\right)$ with polar representation $(F, k)$ has property $\mathscr{P}$ if it satisfies one of the following conditions.

(1) All Darboux points of $F$ are simple and the associated eigenvalues belong to the Morales-Ramis table (By Theorem 2, this condition is equivalent to $\Lambda \subseteq E_{k} \backslash\{k\}$.)

(2) $F$ is finite and nonzero either at the origin, or at infinity (i.e., $k_{0} k_{\infty}=0$ ).

Remark that condition (1) includes the case $F=0$ (since then $\Lambda=$ $\emptyset)$, and that condition (2) includes the case $F$ constant nonzero.

In the case of odd homogeneity degree $k$, the function $F$ is odd due to Proposition 2. Thus the asymptotic exponents $k_{0}, k_{\infty}$ are odd, and so condition (2) of $\mathscr{P}$ cannot occur. Therefore, for odd homogeneity degrees, $\mathscr{P}$ is equivalent to condition (1), which matches exactly the integrability conditions given by [2, Theorem 1] and Theorem 2 .

\subsection{Solving the Diophantine equation}

Equation (11) in Theorem 3 provides a constraint on the possible eigenvalues for a homogeneous potential $V$. We are thus naturally led to study the equation

$$
\sum_{i=1}^{p} \frac{1}{\lambda_{i}-k}=c
$$

where $c$ is a rational number, $k$ the homogeneity degree of $V$, and $p$ an integer related to the number of Darboux points of $V$.

Assume that $V$ is integrable and the assumption of Theorem 3 are satisfied. With our notation, Theorem 2 states that $\Lambda \subseteq E_{k}$, where $E_{k}$ is the set of allowed values in the Morales-Ramis table for degree $k$. Since the relation (11) holds, the aim is to solve equation (12) for unknowns $\lambda_{1}, \ldots, \lambda_{p}$ in $E_{k}$. We will prove that there are only finitely many solutions of this type.

Proposition 4 For any solution $\left(\lambda_{1}, \ldots, \lambda_{p}\right)$ of the equation (12) the following holds

$$
\min \lambda_{i} \leq \frac{p}{c}+k \text { if } c>0, \text { and } \min \lambda_{i} \leq k \text { if } c \leq 0 .
$$

Proof. In the case $c \leq 0$, at least one term in the sum (12) should be negative, and thus $\min \lambda_{i} \leq k$. Let us now look at the case $c>0$. Assume that we have $\lambda_{i}>p / c+k$ for all $1 \leq i \leq p$. Then $\left(\lambda_{i}-k\right)^{-1}<$ $c / p$ and thus $\sum_{i=1}^{p} \frac{1}{\lambda_{i}-k}<c$, which is a contradiction with (12). This proves the proposition.

Let us now remark that all entries of the Morales-Ramis table are bounded below by $\min (0, k)$ (and this minimum is reached for $j=0$ or 1). Starting from this observation, we design a recursive algorithm that finds all the solutions of equation (12) that belong to the MoralesRamis table.

MoralesRamisDiophantineSolve

Input: The parameters $p, k, c$ of the equation (12).

Output: The set of all solutions $\left(\lambda_{1}, \ldots, \lambda_{p}\right)$ in $E_{k}$ of (12), up to permutation.

1. If $p=1$ and $c=0$, then return $\emptyset$. If $p=1$ and $c \neq 0$, then return $1 / c+k$ if it belongs to $E_{k}$, else $\emptyset$. If $p>1$, beginning by $j=0,-1$, compute the elements of $E_{k}$, up to the bound of Proposition 4 . This yields a set $S$.

2. For each entry $S_{i}$ of $S$, recursively run the algorithm on the input $p-1, k, c-1 /\left(S_{i}-k\right)$, with output $R_{i}$.

3. Return the set of solutions $\left[R_{i}, S_{i}\right], i=1 \ldots \sharp S$.

Due to Proposition 4, the equation (12) has finitely many solutions $\left(\lambda_{1}, \ldots, \lambda_{p}\right)$ in $E_{k}$ (this was already proved in [14, Lemma B.1]), and algorithm MoralesRamisDiophantineSolve always terminates. In practice, this algorithm is very costly. The case $k=0, c=1$ with the constraint $\lambda_{i} \in \mathbb{N}$ leads to the equation analysed in [9], for which an optimal bound on $\max \left(\lambda_{1}, \ldots, \lambda_{p}\right)$ is found. This bound is doubly exponential in $p$ (which in our problem is the number of Darboux points). It is natural to conjecture that a similar doubly exponential bound holds in our case.

\section{THE ALGORITHM}

\subsection{Specifications}

Let $\mathbf{a}=\left(\mathbf{a}_{1}, \ldots, \mathbf{a}_{n}\right)$ be parameters and $V \in \mathbb{Q}(i)(\mathbf{a})\left(\mathbf{q}_{1}, \mathbf{q}_{2}\right)$ be a parametrized homogeneous potential. In the sequel, we assume that $V$ is given in canonical form, i.e. the coefficients of its numerator and denominator lie in $\mathbb{Q}[\mathbf{a}]$.

Our goal is to compute a subset $\mathscr{I}(V)$ in the set of parameter values $a$ such that $a \in \mathscr{I}(V)$ is a necessary condition for the integrability of $V\left(a, \mathbf{q}_{1}, \mathbf{q}_{2}\right)$.

In Section 3, we have defined the polar representation of a homogeneous potential with coefficients in $\mathbb{C}$. We can do the same in the context of parametrized homogeneous potential by defining the function 
$F(\mathbf{a}, \mathbf{z})=V\left(\mathbf{a}, \frac{\mathbf{z}+\mathbf{z}^{-1}}{2}, \frac{\mathbf{z}-\mathbf{z}^{-1}}{2 i}\right)$ as in the proof of Proposition 2. With this definition, the following lemma is an immediate consequence of Proposition 2.

Lemma 1 Let $\mathscr{D}$ be the complementary of the common solutions of the coefficients of the denominator of $V$. For all $a \in \mathscr{D},(F(a, \mathbf{z}), k)$ is the polar representation of $V\left(a, \mathbf{q}_{1}, \mathbf{q}_{2}\right)$.

This allows us to define the following set. We let $\mathscr{I}(V)=\mathscr{I}(F, k)$ be the set of values $a$ such that $a \in \mathscr{D}$ and $F(a, \mathbf{z})$ has property $\mathscr{P}$ (Definition 4).

Corollary 1 Let $V \in \mathbb{Q}(\mathbf{a})\left(\mathbf{q}_{1}, \mathbf{q}_{2}\right)$ be a parametrized homogeneous potential, and let $(F, k)$ be its polar representation, $F \in \mathbb{Q}(i)(\mathbf{a})(\mathbf{z})$. If $V\left(a, \mathbf{q}_{1}, \mathbf{q}_{2}\right)$ is integrable, then $a \in \mathscr{I}(F, k)$.

Proof. Assume that $V\left(a, \mathbf{q}_{1}, \mathbf{q}_{2}\right)$ is integrable. Then thanks to [2, Theorem 1], if $F(a, \mathbf{z})$ has a multiple Darboux point, then $F(a, \mathbf{z})$ is constant and thus $a \in \mathscr{I}(F, k)$. If $F(a, \mathbf{z})$ has only simple Darboux points, Theorem 2 implies that eigenvalues at Darboux points are all in $E_{k}$. Thus condition (1) of $\mathscr{P}$ is satisfied, and thus $a \in \mathscr{I}(F, k)$.

Our main algorithm IntegrabilityConditions in Section 5.3 will take as input a parametrized homogeneous potential $V \in \mathbb{Q}(i)(\mathbf{a})\left(\mathbf{q}_{1}, \mathbf{q}_{2}\right)$ and will compute polynomial constraints in $\mathbb{Q}[\mathbf{a}]$ that define the Zariski closure of $\mathscr{I}(V)$.

It uses a subroutine that takes as input special parametrized polar representations $(G, k)$ and computes polynomial constraints that define the intersection of $\mathscr{I}(G, k)$ with the subset of the parameter space over which the valuation and number of roots/poles of $G$ counted with multiplicities is constant.

\subsection{Subroutine for model functions}

Definition 5 We say that $G \in \mathbb{Q}(\mathbf{w})(\mathbf{z})$ is a model function if either $G=0$ identically, or there exist $\alpha \in \mathbb{Z}, \beta_{i} \in \mathbb{N}$ with finitely many non zero $\beta_{i}$ 's, such that

$$
G=\mathbf{w}_{0,0} \mathbf{z}^{\alpha} \prod_{\substack{i \in \mathbb{Z}^{*} \\ \beta_{i}>0}} B_{i}^{i}
$$

with $B_{i}=\mathbf{z}^{\beta_{i}}+\sum_{j=0}^{\beta_{i}-1} \mathbf{w}_{i, j} \mathbf{z}^{j}$. We then denote this function by $G=$ $G_{\alpha, \beta}$; we will write $N_{\alpha, \beta}$ for the number of parameters of $G_{\alpha, \beta}$.

In the following, when there will be no ambiguity on $\alpha, \beta$ (which will be mostly the case), they will be omitted in the subscripts.

Definition 6 Assume $G_{\alpha, \beta} \neq 0$. We let $\Omega_{\alpha, \beta} \subset \mathbb{C}^{N_{\alpha, \beta}}$ be the subset of the parameter space defined by $\Pi\left(G_{\alpha, \beta}\right) \neq 0$ where

$$
\Pi\left(G_{\alpha, \beta}\right)=\mathbf{w}_{0,0} \prod_{\beta_{i}>0} \mathbf{w}_{i, 0} \prod_{\beta_{i}>0} \operatorname{res}\left(B_{i}, B_{i}^{\prime}\right) \prod_{\beta_{i}>0, \beta_{j}>0, j \neq i} \operatorname{res}\left(B_{i}, B_{j}\right)
$$

where $\operatorname{res}\left(A_{1}, A_{2}\right)$ denotes the resultant of two polynomials $A_{1}, A_{2} \in$ $\mathbb{Q}[\mathbf{w}][\mathbf{z}]$ with respect to $\mathbf{z}$.

Remark that for a $w \in \Omega_{\alpha, \beta}$, the roots of the $B_{i}$ 's are all simple and non zero. Moreover, the $B_{i}$ 's do not have any common root.

Definition 7 Assume $G_{\alpha, \beta} \neq 0$. Let $S$ be a finite subset of $\mathbb{Q}$. We define the polynomials in $\mathbb{Q}[\mathbf{w}, \mathbf{z}]$

$$
Z_{1}=\operatorname{numer}\left(\frac{G_{\alpha, \beta}^{\prime}}{G_{\alpha, \beta}}\right), Z_{2, S}=\prod_{\lambda \in S} \operatorname{numer}\left(k-\frac{\mathbf{z}^{2} G_{\alpha, \beta}^{\prime \prime}}{G_{\alpha, \beta}}-\lambda\right),
$$

where numer $(f)$ denotes the numerator of $f$.

The rest of this section is devoted to the design of an algorithm called IntegrabilityConditionsModelFamily, that takes as input a model family $G_{\alpha, \beta}$ and an integer $k$ and returns a set of polynomial equations and inequalities in $\mathbb{Q}[\mathbf{w}]$ that define the intersection of $\Omega_{\alpha, \beta}$ and $\mathscr{I}\left(G_{\alpha, \beta}, k\right)$.

We are now ready to describe our algorithm.

IntegrabilityConditionsModeIFamily

Input: A model family $G_{\alpha, \beta}$ and an integer $k \neq-2,0,2$.

Output: A pair $\left(L, \Pi\left(G_{\alpha, \beta}\right)\right)$ such that $L$ is a list of lists of polynomials $L_{1}, \ldots, L_{\ell}$ and $\Omega_{\alpha, \beta} \cap \mathscr{I}\left(G_{\alpha, \beta}, k\right)$ is defined by the union of the zero-sets of the polynomials in $L_{i}$ and at which $\Pi\left(G_{\alpha, \beta}\right) \neq 0$ for $1 \leq i \leq \ell$.

1. If $G_{\alpha, \beta}=0$ then return $(\emptyset, \emptyset)$.

2. Compute the polynomial $\Pi\left(G_{\alpha, \beta}\right)$.

3. If $\alpha\left(\alpha+\sum_{i \in \mathbb{Z}} i \beta_{i}\right)=0$ then return $\left(\emptyset, \Pi\left(G_{\alpha, \beta}\right)\right)$.

4. Else

(a) Compute the coefficients $c, p$ of the relation $\sum_{j=1}^{p} \frac{1}{\lambda_{j}-k}=c$ with $p=\operatorname{deg}_{\mathbf{z}} Z_{1}(\mathbf{w}, \mathbf{z})$ and $c=1 / \alpha-1 /\left(\alpha+\sum_{i \in \mathbb{Z}} i \beta_{i}\right)$.

(b) Solve this equation using algorithm DiophantineSolve; let $\mathscr{S}$ be its output.

(c) For each solution $S$ in $\mathscr{S}$, build the polynomial $Z_{2, S}$.

(d) Compute the remainder $R_{S}$ for the Euclidean division of $Z_{2, S}$ by $Z_{1}$ in $\mathbb{Q}[\mathbf{w}][\mathbf{z}]$ and let $\mathscr{L}_{S}$ be the sequence of polynomials $R_{i, S}$ for $i \geq 0$.

(e) Let $\mathscr{L}$ be the concatenation of all $\mathscr{L}_{S}$ for $S \in \mathscr{S}$; return $\left(\mathscr{L}, \Pi\left(G_{\alpha, \beta}\right)\right)$.

Before proving the correctness of our algorithm, we will first prove the following two lemmas.

Lemma 2 Assume that $G_{\alpha, \beta} \neq 0, \alpha \neq 0$ and let $w \in \Omega_{\alpha, \beta}$. The set of Darboux points of $G_{\alpha, \beta}(w, \mathbf{z})$ is equal to the set of roots of $Z_{1}(w, \mathbf{z})$. Moreover, if $\zeta$ is a simple Darboux point of $G_{\alpha, \beta}(w, \mathbf{z})$, then $\zeta$ is a simple root of $Z_{1}(w, \mathbf{z})$.

PROOF. Let us first prove that any root of $Z_{1}(w, \mathbf{z})$ is a Darboux point of $G$. Let $\zeta$ be a root of $Z_{1}(w, \mathbf{z})$. We need to prove the following

$$
\zeta \neq 0, G(w, \zeta) \neq 0, G^{\prime}(w, \zeta)=0 .
$$

Consider the logarithmic derivative of $G$

$$
\frac{G^{\prime}}{G}(\mathbf{w}, \mathbf{z})=\frac{\alpha}{\mathbf{z}}+\sum_{\beta_{i}>0} i \frac{B_{i}^{\prime}}{B_{i}} .
$$

Taking the numerator of this expression, we obtain

$$
Z_{1}(\mathbf{w}, \mathbf{z})=\alpha \prod_{\beta_{i}>0} B_{i}+\mathbf{z} \sum_{\beta_{j}>0} j B_{j}^{\prime} \prod_{\beta_{i}>0, i \neq j} B_{i} .
$$

Evaluating this expression at $(\mathbf{w}, \mathbf{z})=(w, 0)$ gives

$$
Z_{1}(w, 0)=\prod_{\beta_{i}>0} w_{i, 0} .
$$

This quantity is non zero as $w \in \Omega$ (due to Definition 6). Thus $\zeta \neq 0$. Let us now prove that any non zero root and pole of $G(w, \mathbf{z})$ is not a root of $Z_{1}(w, \mathbf{z})$. Let $\eta \neq 0$ be a root or a pole of $G$. Then $\eta$ cancels one and only one of the factors $B_{i}$ (let's say $B_{i_{0}}$ ) of $G$ (because for $w \in \Omega$, the $B_{i}$ 's have no common root due to Definition 6). Now evaluating the expression (15) at $\eta$, we obtain

$$
Z_{1}(w, \eta)=\eta i_{0} B_{i_{0}}^{\prime}(w, \zeta) \prod_{\beta_{i}>0, i \neq i_{0}} B_{i}(w, \eta) .
$$

This quantity is non zero because $B_{i_{0}}^{\prime}(w, \eta) \neq 0$ (in Definition 6 , the $B_{i}$ 's have only simple roots). Thus $\eta$ is not a root of $Z_{1}(w, \mathbf{z})$, and therefore $\zeta$ is not a root nor a pole of $G(w, \mathbf{z})$. To conclude, we have

$$
G^{\prime}(\mathbf{w}, \mathbf{z})=G(\mathbf{w}, \mathbf{z}) \frac{Z_{1}(\mathbf{w}, \mathbf{z})}{\mathbf{z} \prod_{\beta_{i}>0} B_{i}(\mathbf{w}, \mathbf{z})} .
$$

The function $G$ is well defined at $(\mathbf{w}, \mathbf{z})=(w, \zeta)$ (i.e. has a finite value), the $B_{i}$ 's do not vanish at $(\mathbf{w}, \mathbf{z})=(w, \zeta)$, and thus $G^{\prime}(w, \zeta)=0$. 
Let us now prove the reverse. If $\zeta$ is a Darboux point of $G(w, \mathbf{z})$, then $G(w, \zeta)$ is finite and non zero, and $G^{\prime}(w, \zeta)=0$. Using equality (16) at $(\mathbf{w}, \mathbf{z})=(w, \zeta)$, we obtain $Z_{1}(w, \zeta)=0$.

Finally, let us look at multiplicity. If $\zeta$ is a simple Darboux point, then $G^{\prime \prime}(w, \zeta) \neq 0$. So we differentiate relation (16) in $\mathbf{z}$ and evaluate it at $(\mathbf{w}, \mathbf{z})=(w, \zeta)$. On the right-hand side, all terms vanish except $G(w, \zeta) \frac{Z_{1}^{\prime}(w, \zeta)}{\zeta \prod_{\beta_{i}>0} B_{i}(w, \zeta)}$. As $\zeta$ is a Darboux point, it is neither a pole of $G$, nor a root of any $B_{i}$, and thus $Z_{1}^{\prime}(w, \zeta) \neq 0$. Therefore, $\zeta$ is a simple root of $Z_{1}(w, \mathbf{z})$.

Lemma 3 Assume that $G_{\alpha, \beta} \neq 0, \alpha \neq 0$ and $\alpha+\sum_{i \in \mathbb{Z}} i \beta_{i} \neq 0$. Let $S$ be a finite set with $k \notin S$ and let $w \in \Omega_{\alpha, \beta}$. The polynomial $Z_{1}(w, \mathbf{z})$ divides $Z_{2, S}(w, \mathbf{z})$ if and only if $\Lambda\left(G_{\alpha, \beta}, k\right) \subset S$ and all Darboux points of $G_{\alpha, \beta}(w, \mathbf{z})$ are simple.

Proof. Let us first assume that $Z_{1}(w, \mathbf{z})$ divides $Z_{2 . S}(w, \mathbf{z})$. Thus all roots of $Z_{1}$ are roots of $Z_{2, S}$. By Lemma 2, the set of roots of $Z_{1}(w, \mathbf{z})$ is the set of Darboux points of $G$. Let $\zeta$ be a Darboux point of $G$. As $Z_{1}(w, \zeta)=0$, we have $Z_{2, S}(w, \zeta)=0$ and thus at least one factor of the product defining $Z_{2, S}(\mathbf{w}, \mathbf{z})$ (Eq. 14) is zero. So there exists $\lambda_{0} \in S$ such that

$$
\text { numer }\left(k-\frac{\mathbf{z}^{2} G^{\prime \prime}(\mathbf{w}, \mathbf{z})}{G(\mathbf{w}, \mathbf{z})}-\lambda_{0}\right)_{(\mathbf{w}, \mathbf{z})=(w, \zeta)}=0 .
$$

We have

$$
k-\frac{\mathbf{z}^{2} G^{\prime \prime}(\mathbf{w}, \mathbf{z})}{G(\mathbf{w}, \mathbf{z})}-\lambda_{0}=\frac{\operatorname{numer}\left(k-\frac{\mathbf{z}^{2} G^{\prime \prime}(\mathbf{w}, \mathbf{z})}{G(\mathbf{w}, \mathbf{z})}-\lambda_{0}\right)}{\operatorname{denom}\left(k-\frac{\mathbf{z}^{2} G^{\prime \prime}(\mathbf{w}, \mathbf{z})}{G(\mathbf{w}, \mathbf{z})}-\lambda_{0}\right)} .
$$

To prove that the left-hand side of this equality equals 0 at $(w, \zeta)$, we only need to prove that the denominator of the right-hand side does not vanish. This denominator is always a product of a power of $\mathbf{z}$, $\mathbf{w}_{0,0}$ and powers of $B_{i}(\mathbf{w}, \mathbf{z})$. If such a product vanishes at $(w, \zeta)$, then exactly one of the $B_{i}$ 's vanishes (as $\zeta \neq 0$ and the $B_{i}$ 's do not have common roots), and then either $\zeta$ is a root or a pole of $G(w, \mathbf{z})$ This is impossible, since $\zeta$ is a Darboux point of $G(w, \mathbf{z})$. Thus $k-$ $\zeta^{2} G^{\prime \prime}(w, \zeta) / G(w, \zeta)=\lambda_{0}$. So the eigenvalue associated to $\zeta$ is $\lambda_{0}$ and it belongs to $S$. Thus $\Lambda(G, k) \subset S$. Moreover, as $k \notin S$, we have $k \notin \Lambda(G, k)$, and by Proposition 3 , this implies that $G^{\prime \prime}(w, \zeta) \neq 0$ and all Darboux points of $G$ are simple.

Conversely, assume that $\Lambda(G, k) \subset S$ and all Darboux points are simple. Let $\zeta$ be a root of $Z_{1}(w, \mathbf{z})$. Then $\zeta$ is a Darboux point, and thus $k-\zeta^{2} G^{\prime \prime}(w, \zeta) / G(w, \zeta) \in \Lambda$. Then there exists $\lambda_{0} \in S$ such that $k-\zeta^{2} G^{\prime \prime}(w, \zeta) / G(w, \zeta)=\lambda_{0}$. Thus numer $\left(k-\frac{\mathbf{z}^{2} G^{\prime \prime}(\mathbf{w}, \mathbf{z})}{G(\mathbf{w}, \mathbf{z})}-\lambda_{0}\right)$ evaluated at $(w, \zeta)$ equals 0 , and so $Z_{2, S}(w, \zeta)=0$. So all roots of $Z_{1}(w, \mathbf{z})$ are roots of $Z_{2, S}(w, \mathbf{z})$. As moreover all roots of $Z_{1}(w, \mathbf{z})$ are simple, $Z_{1}(w, \mathbf{z})$ divides $Z_{2, S}(w, \mathbf{z})$.

Theorem 4 Algorithm IntegrabilityConditionsModelFamily takes a input a model function $G_{\alpha, \beta}$ and returns a set of polynomial constraints that define $\Omega_{\alpha, \beta} \cap \mathscr{I}\left(G_{\alpha, \beta}, k\right)$.

Proof. When $G$ is identically 0, i.e. there is no Darboux point, the set of parameters is $\{\bullet\}=\mathbb{C}^{0}$ and the equalities $\mathscr{I}=\Omega=\{\bullet\}=$ $\mathbb{C}^{0}$ hold by convention. An empty list is returned (Step 1) since there is no parameter. For the rest of the proof, we may assume that $G$ is not 0 identically.

Let us denote by $\mathscr{O}$ the set defined by the output of the algorithm IntegrabilityConditionsModelFamily. Let us first prove that $\mathscr{O} \subset$ $\Omega_{\alpha, \beta} \cap \mathscr{I}\left(G_{\alpha, \beta}, k\right)$. First remark that $\mathscr{O} \subset \Omega$ as the output of IntegrabilityConditionsModelFamily always contains $\Pi\left(G_{\alpha, \beta}\right) \neq 0$.

First case: $\alpha\left(\alpha+\sum_{i \in \mathbb{Z}} i \beta_{i}\right)=0$. The output returned at Step 3 is simply $\Pi(G) \neq 0$, and thus $\mathscr{O}=\Omega$. Remark that when $w \in \Omega$, 0 is not a root of the $B_{i}$ 's. So the asymptotic exponent of $G(w, \mathbf{z})$ at 0 is $k_{0}=\alpha$. At infinity, the degrees of the $B_{i}$ 's are exactly $\beta_{i}$ (as the polynomials are monic). Thus we obtain $k_{\infty}=\alpha+\sum_{i \in \mathbb{Z}} i \beta_{i}$, and we have $k_{0} k_{\infty}=0$, and the second case of Property $\mathscr{P}$ is satisfied. So $\mathscr{P}$ is satisfied. Thus $\mathscr{O} \subset \Omega_{\alpha, \beta} \cap \mathscr{I}\left(G_{\alpha, \beta}, k\right)$.

Second case: $\alpha\left(\alpha+\sum_{i \in \mathbb{Z}} i \beta_{i}\right) \neq 0$. Let $w \in \mathscr{O}$. Thus $w \in \Omega$ (because of the inequality $\Pi(G) \neq 0$ returned at Step 4e). Moreover, there exists a $S \in \mathscr{S}$ such that $Z_{1}(w, \mathbf{z})$ divides $Z_{2 . S}(w, \mathbf{z})$ (as the Step 4e returns the disjunction $\bigvee_{S \subset \mathscr{S}} \mathscr{L}_{S}$ ). The list $S$ belongs to $\mathscr{S}$ thanks to Step $4 \mathrm{c}$ and $\mathscr{S}$ is the output of DiophantineSolve with parameters $p=\operatorname{deg}_{\mathbf{z}} Z_{1}(\mathbf{w}, \mathbf{z}), k, c=1 / \alpha-1 /\left(\alpha+\sum_{i \in \mathbb{Z}} i \beta_{i}\right)$ (Step 4b). Thus $k \notin S$. The hypotheses of Lemma 3 are now satisfied, which gives $\Lambda \subset S$ and all Darboux points of $G$ are simple. As $S \subset E_{k}$, the condition $\Lambda \subset E_{k}$ is satisfied and then so is Property $\mathscr{P}$.

Conversely, let us now prove $\Omega_{\alpha, \beta} \cap \mathscr{I}\left(G_{\alpha, \beta}, k\right) \subset \mathscr{O}$.

First case: $\alpha\left(\alpha+\sum_{i \in \mathbb{Z}} i \beta_{i}\right)=0$. Then we have for the output $\mathscr{O}=\Omega$ (Step 3$)$, and so the inclusion is trivially satisfied.

Second case: $\alpha\left(\alpha+\sum_{i \in \mathbb{Z}} i \beta_{i}\right) \neq 0$. Let $w \in \Omega_{\alpha, \beta} \cap \mathscr{I}\left(G_{\alpha, \beta}, k\right)$. As $w \in \Omega$, the asymptotic exponents of $G(w, \mathbf{z})$ are $k_{0}=\alpha, k_{\infty}=(\alpha+$ $\left.\sum_{i \in \mathbb{Z}} i \beta_{i}\right)$. Thus $k_{0} k_{\infty} \neq 0$, and so the second property of $\mathscr{P}$ is not satisfied. So the first one has to be satisfied (as $w \in \mathscr{I}\left(G_{\alpha, \beta}, k\right)$ ). So all Darboux points of $G$ are simple, and $k_{0} k_{\infty} \neq 0$ : the hypotheses of Theorem 3 are satisfied, and thus the set $\Lambda$ satisfies the relation (11). As moreover $\Lambda \subset E_{k}$ (due to the first property of $\mathscr{P}$ ), the set $\Lambda$ should be up to permutation one (let us say $S_{0}$ ) of the lists of the output $\mathscr{S}$ of the algorithm DiophantineSolve with parameters $c=$ $1 / k_{0}-1 / k_{\infty}, p=\operatorname{deg}_{\mathbf{z}} Z_{1}(w, \mathbf{z})$.

We now need to check that those parameters are indeed those we use in Step 4a. In particular, we have yet to prove that $\operatorname{deg}_{\mathbf{z}} Z_{1}(w, \mathbf{z})=$ $\operatorname{deg}_{\mathbf{z}} Z_{1}(\mathbf{w}, \mathbf{z})$. We use relation (16). As $w \in \Omega$, the asymptotic exponent of $G(w, \mathbf{z})$ at infinity is $\alpha+\sum_{i \in \mathbb{Z}} i \beta_{i} \neq 0$ (and equal to the asymptotic exponent of $G(\mathbf{w}, \mathbf{z}))$. So the asymptotic exponent of $G^{\prime}(w, \mathbf{z}) / G(w, \mathbf{z})$ at infinity is -1 . For any $w$, the degree of the $B_{i}$ 's are $\beta_{i}$. So the asymptotic exponent of $Z_{1}(w, \mathbf{z})$ (its degree) is $-1+1+$ $\sum_{i \in \mathbb{Z}^{*}} \beta_{i}$, which is equal to the one of $Z_{1}(\mathbf{w}, \mathbf{z})$.

Thus the set $\mathscr{S}$ is indeed the one we compute in Step $4 \mathrm{~b}$. So in particular, we have $\Lambda \subset S_{0}$ (seeing now $S_{0}$ as a set). Now using Lemma 3, we obtain that $Z_{1}(w, \mathbf{z})$ divides $Z_{2}, s(w, \mathbf{z})$. Step 4 e returns a sequence of list of polynomials $\mathscr{L}_{1}, \ldots, \mathscr{L}_{\ell}$ such that, for each $S \in \mathscr{S}$ and $w$ in the solution set of some $\mathscr{L}_{i}, Z_{1}(w, \mathbf{z})$ divides $Z_{2, S}(w, \mathbf{z})$. So this condition is satisfied. The inequality $\Pi(G) \neq 0$ is also satisfied as $w \in \Omega$. Thus $w$ satisfies conditions returned by IntegrabilityConditionsModelFamily, and so $w \in \mathscr{O}$.

\subsection{Main algorithm}

We are now ready to present our main algorithm IntegrabilityConditions. It uses some basic operations on ideals of polynomial rings. If $I, J$ are ideals, $I: J^{\infty}$ denotes the saturated ideal $\{f \mid \exists g \in J, \exists N \in$ $\left.\mathbb{N}, f g^{N} \in I\right\}$. We refer to [5, Chap 2. Sec. 1] for more details.

IntegrabilityConditions

Input: $V \in \mathbb{Q}(i)(\mathbf{a})\left(\mathbf{q}_{1}, \mathbf{q}_{2}\right) k$-homogeneous, given in canonical form (i.e. the coefficients of numerator / denominator of $V$ lie in $\mathbb{Q}[\mathbf{a}]$ ).

Output: A sequence of sets of polynomials $H_{1}, \ldots, H_{\ell}$ in a such that the union of the common complex solutions of $H_{i}$ defines the Zariski closure of $\mathscr{I}(V)$.

1. Write $V$ in polar coordinates $V\left(\mathbf{a}, q_{1}, q_{2}\right)=r^{k} F\left(\mathbf{a}, e^{i \theta}\right)$.

2. Let $\Delta$ be the sequence of coefficients of the denominator of $V$.

3. Compute all the model functions $G_{\alpha, \beta}$ whose numerator and denominator have degrees in $z$ less than those of $F$.

4. For all possible $(\alpha, \beta)$, let $\left(\mathscr{L}_{\alpha, \beta}, W_{\alpha, \beta}\right)$ be the output of IntegrabilityConditionsModelFamily with input $G_{\alpha, \beta}$ and $k$.

5. Let $\mathscr{C}_{\alpha, \beta}$ be the list of coefficients in $\mathbf{z}$ of the numerator of $F-G_{\alpha, \beta}$.

6. For each family $G_{\alpha, \beta}$ and for all lists $\mathscr{L}$ in $\mathscr{L}_{\alpha, \beta}$, compute a set $H_{\alpha, \beta}(\mathscr{L})$ of generators of the elimination ideal in a of the ideal generated by

$$
\left\langle\mathscr{L}, W_{\alpha, \beta} T-1, \mathscr{C}_{\alpha, \beta}\right\rangle:\langle\Delta\rangle^{\infty} \cap \mathbb{Q}(i)[\mathbf{a}] .
$$


7. Return the list of all sets $H_{\alpha \beta}(\mathscr{L})$.

Below, we reuse the notation $\mathscr{D}$ for the non-empty Zariski open set introduced in Lemma 1.

Theorem 5 Let $V \in \mathbb{Q}(i)(\mathbf{a})\left(\mathbf{q}_{1}, \mathbf{q}_{2}\right)$ be a k-homogeneous potential. Algorithm IntegrabilityConditions takes as input $V$ and returns polynomial conditions defining the Zariski closure of $\mathscr{I}(V)$.

Proof. In the sequel, we denote by $\overline{\mathscr{I}(V)}$ the Zariski closure of $\mathscr{I}(V)$ and we let $\mathscr{O}$ be the set defined by the output of Algorithm IntegrabilityConditions. Note that since this set is an algebraic set, it is closed. We prove below that $\mathscr{O}=\overline{\mathscr{I}(V)}$.

Take $a \in \mathscr{I}(V)$; we prove below that $a \in \mathscr{O}(V)$ from which we deduce that $\mathscr{I}(V) \subset \mathscr{O}$. Since $\mathscr{O}$ is closed for the Zariski topology, we conclude that $\overline{\mathscr{I}(V)} \subset \mathscr{O}$. Recall that, by assumption, $a \in$ $\mathscr{I}(V)=\mathscr{I}(F, k)$; then $a \in \mathscr{D}$ and $F(a, \mathbf{z})$ has property $\mathscr{P}$ (see Definition 4). We let $\alpha$ be the valuation of $F(a, \mathbf{z})$ and $\beta_{i}$ be the number of roots/poles of multiplicity $i$. Thus, we consider the model function $G_{\alpha, \beta}$; for $w \in \Omega_{\alpha, \beta}, G_{\alpha, \beta}(w, \mathbf{z})$ has the same features as those of $F(a, \mathbf{z})$. This implies that $\Omega_{\alpha, \beta}$ contains a common root to the polynomials in $\mathscr{C}_{\alpha, \beta}$ (Step 6) obtained after instantiating a to $a$; let $w$ be one of these roots.

Recall that $\mathscr{C}_{\alpha, \beta}$ is the list of coefficients of the numerator of $F-$ $G_{\alpha, \beta}$ (seen as a polynomial in $\mathbb{C}[\mathbf{z}]$ ). Also, since $a \in \mathscr{D}$, the denominator of $F(a, \mathbf{z})$ is not identically 0 and we deduce that $F(a, \mathbf{z})=$ $G_{\alpha, \beta}(w, \mathbf{z})$. Note that $w \in \Omega_{\alpha, \beta}$ by construction; also, by assumption, $a \in \mathscr{I}(V)$, and thus $F(a, \mathbf{z})=G_{\alpha, \beta}(w, \mathbf{z})$ has property $\mathscr{P}$. We deduce that $w \in \mathscr{I}\left(G_{\alpha, \beta}, k\right)$. Thus, correctness of Algorithm IntegrabilityConditionsModelFamily (Theorem 4) implies that $w$ is a solution of the systems output at Step 4 of the main algorithm. Now, by construction $(w, a)$ is not a common solution of the polynomials in $\Delta$ and is a solution of the system obtained by setting to 0 the polynomials in $\mathscr{C}_{\alpha, \beta}, \mathscr{L}$ and the inequalities $W_{\alpha, \beta} \neq 0$, for some $\mathscr{L}$ in $\mathscr{L}_{\alpha, \beta}$. Thus, the fact that $H_{\alpha, \beta}$ vanishes at $a$ is immediate and we conclude that $a \in \mathscr{O}$ as requested.

To finish the proof, we establish that $\mathscr{O} \subset \overline{\mathscr{I}(V)}$; take $a^{\prime} \in \mathscr{O}$. Then, there exists a set of polynomial equations $H_{\alpha, \beta}$ at Step 6 that is satisfied by $a^{\prime}$. Let $G_{\alpha, \beta}$ be a model function associated to $(\alpha, \beta)$ (note that $(\alpha, \beta)$ may be non unique).

By Theorem 4, the call to IntegrabilityConditionsModelFamily at Step 4 returns a set of polynomial equations and inequalities that define $\Omega_{\alpha, \beta} \cap \mathscr{I}\left(G_{\alpha, \beta}, k\right)$. Reusing the notations of Step 4 b, let $\mathscr{U}_{\alpha, \beta}$ be the constructible set defined by $W_{\alpha, \beta} \neq 0$, the vanishing of all polynomials in $\mathscr{L}$ (for some $\mathscr{L}$ in $\mathscr{L}_{\alpha, \beta}$ ) and the non-vanishing of at least one polynomial in $\Delta$.

Let $\mathscr{A}_{\alpha, \beta}$ be the projection of $\mathscr{U}_{\alpha, \beta}$ on the a-space; since $\mathscr{U}_{\alpha, \beta}$ is a constructible set, $\mathscr{A}_{\alpha, \beta}$ is a constructible set [4, Chap. 3 Sect. 2]. By the elimination theorem [4, Chap. 3 Theorem 2], the set of equations $H_{\alpha, \beta}$ defines the Zariski closure of $\mathscr{A}_{\alpha, \beta}$.

Lemma 4 Let $(w, a) \in \mathscr{U}_{\alpha, \beta}$. Then $w \in \Omega_{\alpha, \beta} \cap \mathscr{I}\left(G_{\alpha, \beta}, k\right)$ and $a \in$ $\mathscr{I}(F, k)$.

PROOF. By assumption, $w$ is a solution of the output of IntegrabilityConditionsModelFamily performed with input $G_{\alpha, \beta}$ and $k$. The fact that $w \in \Omega_{\alpha, \beta} \cap \mathscr{I}_{\alpha, \beta}$ is a direct consequence of Theorem 4 that states the correctness of IntegrabilityConditionsModelFamily. Since $w \in \Omega_{\alpha, \beta} \cap \mathscr{I}\left(G_{\alpha, \beta}, k\right)$, we deduce that $\left(G_{\alpha, \beta}(w, \mathbf{z}), k\right)$ has property $\mathscr{P}$ (see Definition 4 ).

Now, remark that for all $(w, a) \in \mathscr{U}_{\alpha, \beta}, F(a, \mathbf{z})=G_{\alpha, \beta}(w, \mathbf{z})$ (because the equations in $\mathscr{C}_{\alpha, \beta}$ are satisfied; see Step 6). We deduce that $F(a, \mathbf{z})$ has the property $\mathscr{P}$; and hence $a \in \mathscr{I}(F, k)=\mathscr{I}(V)$.

By Lemma 4, we conclude that $\mathscr{A}_{\alpha, \beta} \subset \mathscr{I}(F, k)$ which, by definition 4 , is $\mathscr{I}(V)$. Then, the Zariski closure of $\mathscr{A}_{\alpha, \beta}$ is contained in the Zariski closure of $\mathscr{I}(F, k)=\mathscr{I}(V)$. Since $\mathscr{O}$ is the union of the
Zariski closures of $\mathscr{A}_{\alpha \beta}$ for all possible $(\alpha, \beta)$, we deduce that $\mathscr{O}$ is contained in the Zariski closure of $\mathscr{I}(V)$. This concludes the proof.

\section{EXPERIMENTAL RESULTS}

The algorithm IntegrabilityConditions provides a general framework for computing necessary conditions for integrability of homogeneous planar potentials. By incorporating more specific integrability criteria (order 2 conditions [22], diagonalizability of Hessians [6], improper Darboux points [28]), the algorithm can be enhanced. This is actually what we implemented, in the computer algebra system Maple (v17). ${ }^{1}$ The main tools that we use involve polynomial ideals, notably relying on the Gröbner engine FGb, implemented by J.-C. Faugère [7] for elimination ideal computation. This step is a crucial one for efficiency. Our implementation succeeds in dealing with around 10 Darboux points for simple enough potentials (typically polynomial potentials), and around 5 in all cases.

Using this implementation, we have been able to provide the first complete proof of the non-integrability of the collinear three body problem, and even of a generalization with electrical interactions (see Theorem 6 below). This implementation is also able to automatically reprove the results in [14] about polynomial potentials of degree at most 4, and, more importantly, to explore polynomial and inverses of polynomial potentials with higher degree (up to 9) leading to the discovery of several new candidates for integrability that were, to our knowledge, previously unknown.

Collinear three body problem. Some classical dynamical problems, such as the collinear three body problem (and its generalization to any homogeneity degree), can be written as planar homogeneous potentials, of the form (18) below. For them, our algorithm is able to perform a complete integrability analysis. Previous works on the integrability of the three body problem treated either its simpler planar version $[1,29,23]$, or the collinear version itself, but under restrictive assumptions [32, 21, 27]. For instance, non-integrability was proven by Yoshida [32] and Morales and Ramis [21] in the case of equal masses; Shibayama [27, Theorem 3] considered the case of arbitrary masses, but his proof is valid only for the classical collinear three body problem, and does not take into account some exceptional cases. Our algorithm proves the following complete classification result.

Theorem 6 The problem of three bodies interacting pairwise by a force proportional to the inverse of the square of the mutual distance, after reduction by translation, is not integrable.

To prove this result, we first observe that the three body problem can be rewritten as a potential of the form

$$
V\left(\mathbf{q}_{1}, \mathbf{q}_{2}\right)=\mathbf{a q} \mathbf{q}_{1}+\mathbf{b} \mathbf{q}_{2}^{-1}+\mathbf{c} \mathbf{q}_{1}+\mathbf{d} \mathbf{q}_{2}^{-1}+\mathbf{e q} \mathbf{q}_{1}+\mathbf{f} \mathbf{q}_{2}{ }^{-1} .
$$

It is only necessary to study its integrability up to rotation-dilatation, and thus after reparametrization, we can reduce the problem to the following function $F(\mathbf{z})=\mathbf{z} /\left(\mathbf{a z}^{2}+\mathbf{b}\right)+\mathbf{z} /\left(\mathbf{c z}^{2}+\mathbf{d}\right)+\mathbf{z} /\left(\mathbf{z}^{2}+1\right)$. Our algorithm (after simplification) produces the following integrability conditions on the parameters

$$
\begin{array}{r}
{[\mathbf{b}-\mathbf{a}, \mathbf{d}-\mathbf{c}],[\mathbf{b}-\mathbf{a}, \mathbf{c}+\mathbf{d}],[\mathbf{a}+1, \mathbf{b}+1],[\mathbf{a}+\mathbf{b}, \mathbf{d}-\mathbf{c}],} \\
{[\mathbf{a}+\mathbf{b}, \mathbf{c}+\mathbf{d}],[\mathbf{a}+\mathbf{c}, \mathbf{d}+\mathbf{b}],[\mathbf{c}+1, \mathbf{d}+1] .}
\end{array}
$$

In all the above cases, the function $F$ simplifies to the form $F(\mathbf{z})=$ $\mathbf{z} /\left(\alpha \mathbf{z}^{2}+\beta\right)+\mathbf{z} /\left(\gamma \mathbf{z}^{2}+\delta\right)$. The three body problem potential is a sum of three interactions, which have a singularity when two bodies collapse. The above functions $F$ have only two singularities instead of three, and so at least two bodies do not interact (as this would lead to a singularity). This finishes the proof of Theorem 6 . This result is here proved for the first time for the most general form of the potential.

${ }^{1}$ Our Maple implementation can be downloaded at the url http: //combot.perso.math. cnrs. fr/software.html. 
Polynomials and inverses of polynomials. These potentials are very simple; they have been studied extensively in $[11,12,13$, $8,24]$, and contain some interesting integrable potentials. For our algorithm, these potentials are also simpler than typical rational ones, because they do not involve simplifications between numerators and denominators. Thus fewer functions $G_{\alpha, \beta}$ need to be analyzed. Below we reproduce some results of non-integrability of homogeneous polynomial potentials and inverses of homogeneous polynomial potentials, and we extend them to higher degrees than previously known thanks to our algorithm (the positive degree corresponding to a polynomial, and a negative degree to an inverse of a polynomial). A polynomial homogeneous potential leads to a function $F$ of the form $F(\mathbf{z})=\sum_{i=0}^{k} a_{i} \mathbf{z}^{k-2 i}$ (respectively $F(\mathbf{z})=1 / \sum_{i=0}^{k} a_{i} \mathbf{z}^{k-2 i}$ for the inverse of a homogeneous polynomial). Below we give the sets of eigenvalues for candidates for integrability, and corresponding computation timings.

\begin{tabular}{|c|c|c|}
\hline$k$ & Eigenvalues (the set $\Lambda(F, k)$ in Eq. (9)) & timings \\
\hline 3 & $\{0,6\},\{1,15\},\left\{45, \frac{3}{8}\right\},\{1,10,45\}$ & $0.43 \mathrm{~s}$ \\
\hline 4 & $\{0,12\},\left\{24, \frac{3}{2}\right\},\left\{12,84, \frac{3}{2}\right\},\left\{544, \frac{3}{2}, \frac{35}{2}\right\}$ & $0.91 \mathrm{~s}$ \\
\hline 5 & $\left\{\frac{27}{8}, 135\right\},\left\{\frac{7}{8}, 35,170,665\right\},\{0,20\},\{2,35\}$ & $433 \mathrm{~s}$ \\
\hline 6 & $\{0,30\},\left\{48, \frac{5}{2}\right\}$ & $2.5 \mathrm{~s}$ \\
\hline 7 & $\{0,42\},\{63,3\}$ & $33.5 \mathrm{~s}$ \\
\hline 9 & $\{4\},\{0,72\},\{4,99\}$ & $106 \mathrm{~s}$ \\
\hline-3 & $\{-2\},\{0\},\{3\},\{3,7,12\}$ & $0.36 \mathrm{~s}$ \\
\hline-4 & $\{8,20\},\{0\}$ & $0.639 \mathrm{~s}$ \\
\hline-5 & $\left\{130,-\frac{13}{8}\right\},\{-3,15\},\left\{22,-\frac{13}{8}\right\},\{0\},\{15\}$ & $3.167 \mathrm{~s}$ \\
\hline-6 & $\{24\},\{0\}$ & $594 s$ \\
\hline
\end{tabular}

For polynomials of degree 3 and 4, we retrieve known results (leading to a complete classification of integrable homogeneous potentials of degree 3, and almost complete for degree 4). For $k=4$, we obtain after simplification the following ideals

$$
\begin{array}{r}
{\left[\mathbf{a}_{1}, \mathbf{a}_{2}\right],\left[\mathbf{a}_{4}, \mathbf{a}_{5}\right],\left[36 \mathbf{a}_{5} \mathbf{a}_{1}-\mathbf{a}_{3}^{2}, 6 \mathbf{a}_{4} \mathbf{a}_{1}-\mathbf{a}_{3} \mathbf{a}_{2}, 6 \mathbf{a}_{2} \mathbf{a}_{5}-\mathbf{a}_{4} \mathbf{a}_{3}\right],\left[44979 \mathbf{a}_{2}^{2}-\right.} \\
376712 \mathbf{a}_{3} \mathbf{a}_{1}, 66879684 \mathbf{a}_{5} \mathbf{a}_{1}-75625 \mathbf{a}_{3}^{2}, 16719921 \mathbf{a}_{4} \mathbf{a}_{2}-4708900 \mathbf{a}_{3}{ }^{2}, \\
\left.-376712 \mathbf{a}_{3} \mathbf{a}_{5}+44979 \mathbf{a}_{4}{ }^{2}, 8178 \mathbf{a}_{4} \mathbf{a}_{1}-275 \mathbf{a}_{3} \mathbf{a}_{2}, 8178 \mathbf{a}_{2} \mathbf{a}_{5}-275 \mathbf{a}_{4} \mathbf{a}_{3}\right], \\
{\left[-392 \mathbf{a}_{3} \mathbf{a}_{1}+99 \mathbf{a}_{2}{ }^{2}, 484 \mathbf{a}_{5} \mathbf{a}_{1}-\mathbf{a}_{3}{ }^{2}, 1089 \mathbf{a}_{4} \mathbf{a}_{2}-196 \mathbf{a}_{3}{ }^{2},\right.} \\
\left.-392 \mathbf{a}_{3} \mathbf{a}_{5}+99 \mathbf{a}_{4}{ }^{2}, 22 \mathbf{a}_{4} \mathbf{a}_{1}-\mathbf{a}_{3} \mathbf{a}_{2}, 22 \mathbf{a}_{2} \mathbf{a}_{5}-\mathbf{a}_{4} \mathbf{a}_{3}\right], \\
{\left[-40 \mathbf{a}_{3} \mathbf{a}_{1}+7 \mathbf{a}_{2}{ }^{2}, 15876 \mathbf{a}_{5} \mathbf{a}_{1}-25 \mathbf{a}_{3}{ }^{2}, 441 \mathbf{a}_{4} \mathbf{a}_{2}-100 \mathbf{a}_{3}{ }^{2},\right.} \\
\left.-40 \mathbf{a}_{3} \mathbf{a}_{5}+7 \mathbf{a}_{4}^{2}, 126 \mathbf{a}_{4} \mathbf{a}_{1}-5 \mathbf{a}_{3} \mathbf{a}_{2}, 126 \mathbf{a}_{2} \mathbf{a}_{5}-5 \mathbf{a}_{4} \mathbf{a}_{3}\right]
\end{array}
$$

The first two cases are the exceptional ones with $k_{0}(F) k_{\infty}(F)=0$, and the other cases lead indeed to integrable potentials. These are exactly the conditions found in [14]. At degree 5, two non-trivial new potentials (up to conjugation and rotation-dilatation) are detected, not known to be integrable, but satisfying all integrability conditions. Their eigenvalue sets are $\{27 / 8,135\},\{7 / 8,35,170,665\}$. The second has algebraic coefficients of degree 12 , and the first is

$$
\begin{array}{r}
V\left(\mathbf{q}_{1}, \mathbf{q}_{2}\right)=\left(\mathbf{q}_{1}+i \mathbf{q}_{2}\right)^{2}\left(11391716 i \mathbf{q}_{2}^{3}+73950132 \mathbf{q}_{1} \mathbf{q}_{2}^{2}-\right. \\
\left.150075213 i \mathbf{q}_{1}^{2} \mathbf{q}_{2}-96733564 \mathbf{q}_{1}^{3}\right) .
\end{array}
$$

At degree 6 and 7, the only possible cases are either already known, or $k_{0}(F) k_{\infty}(F)=0$, or they do not have Darboux points. Thus for degree 7 , the only cases whose integrability status is still unknown are up to rotation-dilatation $F(\mathbf{z})=\mathbf{z}$ and $F(\mathbf{z})=\mathbf{z}^{3}$. So we obtained a classification of integrable homogeneous polynomial potentials of degree 7. For degree 8 and 9, some optimizations are necessary for the algorithm to be workable. Indeed, thanks to the fact that our family is invariant by rotation-dilatation, it is only necessary to consider functions $G_{\alpha, \beta}$ with the coefficient $w_{0,0}=1$ and with the trailing coefficient of one polynomial factor equal to 1 . This removes two variables in the elimination ideal, and reduces by 2 the Hilbert dimension of the output. At degree 9, we find three new cases satisfying all integrability conditions; they are given by $F(\mathbf{z})=\mathbf{z}+\mathbf{z}^{-5}, F(\mathbf{z})=$ $\mathbf{z}^{3}+\mathbf{z}^{-3}, F(\mathbf{z})=\mathbf{z}^{-5}\left(\mathbf{z}^{2}+1\right)^{5}$

\section{REFERENCES}

ilité du problème plan des trois corps de masses égales. C. R. Acad. Sci. Paris Sér. I Math., 331(5):391-394, 2000.

[2] T. Combot. Meromorphically integrable homogeneous potentials with multiple Darboux points. Preprint http://arxiv.org/abs/1301.6621.

[3] T. Combot and C. Koutschan. Third order integrability conditions for homogeneous potentials of degree -1. J. Math. Phys., 53(8):Paper \#082704, 26 pp., 2012.

[4] D. Cox, J. Little, and D. O'Shea. Ideals, varieties, and algorithms: an introduction to computational algebraic geometry and commutative algebra. Springer Verlag, 1(5):7-3, 1992

[5] W. Decker and C. Lossen. Computing in algebraic geometry, volume 16. Springer, 2006.

[6] G. Duval and A. J. Maciejewski. Jordan obstruction to the integrability of Hamiltonian systems with homogeneous potentials. Ann. Inst. Fourier (Grenoble), 59(7):2839-2890, 2009.

[7] J.-C. Faugère. FGb. http://www-polsys.lip6.fr/ jcf.

[8] J. Hietarinta. A search for integrable two-dimensional Hamiltonian systems with polynomial potential. Phys. Lett. A, 96(6):273-278, 1983.

[9] O. D. Kellogg. On a Diophantine Problem. Amer. Math. Monthly, 28(8-9):300-303, 1921.

[10] T. Kimura. On Riemann's equations which are solvable by quadratures. Funkcial. Ekvac., 12:269-281, 1969/1970.

[11] J. Llibre, A. Mahdi, and C. Valls. Analytic integrability of Hamiltonian systems with a homogeneous polynomial potential of degree 4. J. Math. Phys. 52(1):012702, 9, 2011.

[12] J. Llibre, A. Mahdi, and C. Valls. Polynomial integrability of the Hamiltonian systems with homogeneous potential of degree -3. Phys. D, 240(24):1928-1935, 2011.

[13] A. J. Maciejewski and M. Przybylska. All meromorphically integrable 2D Hamiltonian systems with homogeneous potential of degree 3. Phys. Lett. A, 327(5-6):461-473, 2004.

[14] A. J. Maciejewski and M. Przybylska. Darboux points and integrability of Hamiltonian systems with homogeneous polynomial potential. J. Math. Phys., 46(6):062901, 33, 2005.

[15] A. J. Maciejewski, M. Przybylska, and H. Yoshida. Necessary conditions for super-integrability of Hamiltonian systems. Phys. Lett. A, 372(34):5581-5587, 2008.

[16] A. J. Maciejewski, M. Przybylska, and H. Yoshida. Necessary conditions for classical super-integrability of a certain family of potentials in constant curvature spaces. J. Phys. A, 43(38):382001, 15, 2010.

[17] A. J. Maciejewski, M. Przybylska, and H. Yoshida. Necessary conditions for the existence of additional first integrals for Hamiltonian systems with homogeneous potential. Nonlinearity, 25(2):255-277, 2012.

[18] R. Martínez and C. Simó. Non-integrability of the degenerate cases of the swinging Atwood's machine using higher order variational equations. Discrete Contin. Dyn. Syst., 29(1):1-24, 2011

[19] J. J. Morales-Ruiz and J. P. Ramis. Galoisian obstructions to integrability of Hamiltonian systems. I. Methods Appl. Anal., 8(1):33-95, 2001.

20] J. J. Morales-Ruiz and J. P. Ramis. Galoisian obstructions to integrability of Hamiltonian systems. II. Methods Appl. Anal., 8(1):97-111, 2001.

21] J. J. Morales-Ruiz and J. P. Ramis. A note on the non-integrability of some Hamiltonian systems with a homogeneous potential. Methods Appl. Anal. $8(1): 113-120,2001$

[22] J. J. Morales-Ruiz, J.-P. Ramis, and C. Simó. Integrability of Hamiltonian systems and differential Galois groups of higher variational equations. Ann. Sci. École Norm. Sup. (4), 40(6):845-884, 2007.

[23] J. J. Morales-Ruiz and S. Simon. On the meromorphic non-integrability of some $N$-body problems. Discrete Contin. Dyn. Syst., 24(4):1225-1273, 2009.

[24] K. Nakagawa and H. Yoshida. A list of all integrable two-dimensional homogeneous polynomial potentials with a polynomial integral of order at most four in the momenta. J. Phys. A, 34(41):8611-8630, 2001.

[25] M. Przybylska. Finiteness of integrable $n$-dimensional homogeneous polynomial potentials. Phys. Lett. A, 369(3):180-187, 2007.

[26] M. E. Sansaturio, I. Vigo-Aguiar, and J. M. Ferrándiz. Non-integrability of some Hamiltonian systems in polar coordinates. J. Phys. A, 30(16):5869-5876, 1997.

[27] M. Shibayama. Non-integrability of the collinear three-body problem. Discrete Contin. Dyn. Syst., 30(1):299-312, 2011.

[28] M. Studziński and M. Przybylska. Darboux points and integrability analysis of Hamiltonian systems with homogeneous rational potentials. Phys. D, 249:1-15, 2013.

[29] A. Tsygvintsev. The meromorphic non-integrability of the three-body problem. $J$. Reine Angew. Math., 537:127-149, 2001.

[30] M. I. Vigo-Aguiar, M. E. Sansaturio, and J. M. Ferrándiz. Integrability of Hamiltonians with polynomial potentials. J. Comput. Appl. Math., 158(1):213-224, 2003. Selected papers from the Conference on Computational and Mathematical Methods for Science and Engineering (Alicante, 2002).

[31] V. Weispfenning. Comprehensive Gröbner bases. J. Symbolic Comput., 14(1):1-29, 1992. 
[32] H. Yoshida. A criterion for the nonexistence of an additional integral in

Hamiltonian systems with a homogeneous potential. Phys. D, 29(1-2):128-142,
1987. 